> Une étude portant sur 51 sujets, dont 15 hommes hémizygotes 12 femmes hétérozygotes et 24 témoins, décrit une méthode de dépistage par dosage de l' $\alpha$-galactosidase A sur goutte de sang recueillie sur papier filtre, et son intérêt dans les populations à risque de maladie de Fabry. <

\section{Avancées récentes dans le dépistage de la maladie de Fabry pour les populations à risque}

Éric Caudron, Diane Molière, Jiang-Yan Zhou, Patrice Prognon, Dominique P. Germain
Ce papier est dédié par Dominique Paul Germain à la mémoire du Professeur Nestor A. Chamoles $\left({ }^{\dagger} 2005\right)$, pionnier du dépistage des maladies lysosomales sur papier filtre.
E. Caudron, J.y. Zhou, P. Prognon : Groupe de Chimie Analytique de Paris Sud, EA 3343, Laboratoire de Chimie Analytique, Faculté de Pharmacie, 5, rue Jean Baptiste Clément, 92296 ChâtenayMalabry, France.

D. Molière : Unité Fonctionnelle de Génétique Clinique, Hôpital Européen Georges Pompidou, 20, rue Leblanc, 75015 Paris, France. D.P. Germain : Groupe de Chimie Analytique de Paris Sud, عA 3343, Laboratoire de Chimie Analytique, Faculté de Pharmacie, 5 , rue Jean Baptiste Clément, 92296 ChâtenayMalabry, France et Unité Fonctionnelle de Génétique Clinique, Hôpital Européen Georges Pompidou, 20, rue Leblanc, 75015 Paris, France. dominique.germain@egp.aphp.fr dent vasculaire cérébral avant 40 ans en l'absence d'étiologie documentée) dans lesquelles la prévalence de la maladie pourrait s'avérer plus élevée que précédemment admis.

\section{Dépistage et diagnostic des femmes hétérozygotes}

La majorité des études de dépistage de la maladie de Fabry ont été réalisées sur des populations d'hommes pour lesquels la mesure de l'activité enzymatique, à partir du sang total déposé sur papier filtre, permet d'établir, sans ambiguïté, un diagnostic de la maladie [1]. En revanche, les hétérozygotes peuvent avoir des activités enzymatiques très variables, en fonction de l'inactivation aléatoire du chromosome $X$ et une activité enzymatique normale chez une femme n'ex- clut donc pas formellement l'absence de maladie [2]. À ce jour, il est difficile d'estimer le pourcentage de femmes porteuses de la mutation, ayant une activité enzymatique normale. Une seule étude pilote portant sur 21 hétérozygotes connues, et réalisée par la mesure de l'activité enzymatique sur papier filtre, a permis de diagnostiquer 13 d'entre elles (62\%) [3]. Aujourd'hui, la seule technique permettant le diagnostic de toutes les hétérozygotes est le test de génotypage du gène GLA qui n'est pas réalisable pour de larges cohortes. Dans ce travail, nous avons souhaité vérifier dans quelle mesure le dosage de l'activité enzymatique à partir d'une goutte de sang déposée sur papier filtre pouvait être adapté au dépistage des femmes hétérozygotes pour la maladie de Fabry sur des populations importantes. 


\section{Quelle est la meilleure technique de dépistage?}

Il apparaît nécessaire de posséder la technique la plus adaptée pour réaliser le dépistage des hémizygotes et d'un maximum d'hétérozygotes tout en connaissant les limites de la technique pour ces femmes hétérozygotes. Dans une optique de dépistage à grande échelle, cette technique devra être facile à mettre en œuvre sur le plan logistique (envois groupés des prélèvements...). Le prélèvement doit être simple et rapide, réalisable par un préleveur non spécifiquement formé. La nature du prélèvement biologique doit permettre une bonne conservation des enzymes (transport vers le laboratoire de référence...). Au niveau du laboratoire, la méthode analytique doit être simple, sensible, spécifique, robuste et automatisable (analyses en série...).

Les techniques envisageables pour des études dans des populations à risques sont nombreuses. La méthode de référence utilisée pour le diagnostic des hémizygotes est le dosage de l'activité enzymatique leucocytaire ou plasmatique [4]. Elle n'a été utilisée en dépistage que sur de petites populations car, d'une part, elle nécessite un acheminement rapide de sang total frais au laboratoire, d'autre part cette technique est assez longue et fastidieuse et n'est donc pas applicable au dépistage à grande échelle. Des travaux plus récents utilisent un support original qui est un papier filtre poreux sur lequel une goutte de sang est déposée $[1,3,5]$. Le prélèvement est extrêmement simple à réaliser et la conservation de l'échantillon est remarquable puisqu'elle est de plusieurs mois à température ambiante [1]. Cette technique est donc parfaitement adaptée à des études de dépistage sur de larges cohortes car la gestion de l'échantillon biologique est très flexible. Autre avantage, cette technique peu coûteuse peut être miniaturisée et automatisée. Un disque du papier filtre, de diamètre connu et correspondant à une certaine quantité de sang total, est incubé à $37^{\circ} \mathrm{C}$, au pH physiologique de l'enzyme et en présence de 4 -méthyl umbelliféryl- $\alpha$-galactopyranoside qui libère le 4-méthyl umbelliférone, molécule fluorescente (4-MU). La réaction est classiquement stoppée par un tampon alcalin à un pH auquel l'enzyme lysosomale est non fonctionnelle. Toutefois, cette technique peut conduire à des résultats erronés en raison d'une interférence liée à la matrice biologique. Cette interférence spectroscopique correspond à une

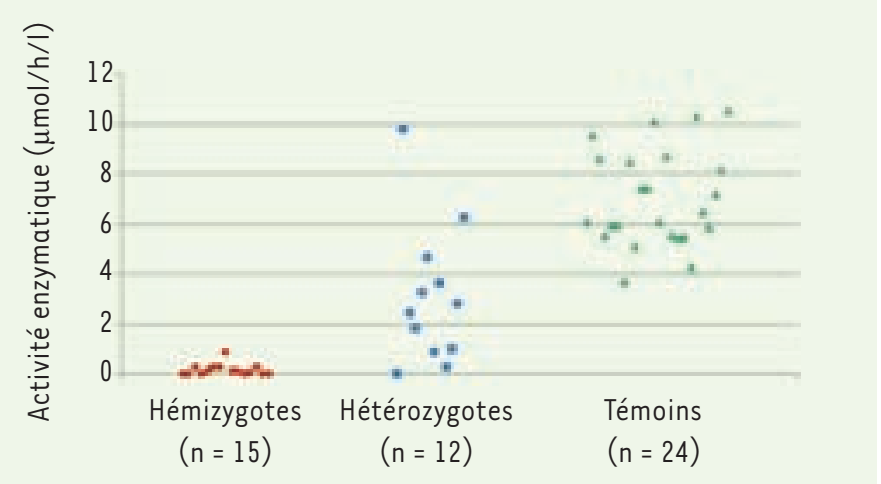

Figure 1. Activité enzymatique $\alpha$-galactosidase $A$ chez 51 patients (Caudron $\varepsilon$, Zhou JY, Prognon P, Germain DP, données non publiées). absorption de la lumière d'excitation et d'émission de fluorescence par les molécules de la matrice biologique. Une étude spectrale dans l'UV-visible de la solution finale sur laquelle la mesure de fluorescence a été réalisée et le spectre obtenu présente une large bande d'absorption dont le maximum d'absorption se situe à $405 \mathrm{~nm}$. Cette bande est attribuée à la bande de Soret, caractéristique du noyau tétrapyrrolique de l'hème, constituant de l'hémoglobine. L'intensité de cette bande est très variable en fonction des patients et présente un recouvrement spectral plus ou moins important au niveau du maximum d'excitation et d'émission du 4-MU. Pour cette raison, nous avons modifié la technique (Caudron $\varepsilon$, Zhou Jy, Prognon P, Germain DP, données non publiées) afin de nous affranchir du quenching de la fluorescence par l'hémoglobine. Le principe est d'éliminer les interférents de nature protéique (hémoglobine) par précipitation à l'éthanol à la fin de la période d'incubation de l'enzyme et du substrat artificiel. Cette précipitation présente le double avantage d'éliminer l'hémoglobine de la matrice biologique mais aussi d'arrêter la réaction enzymatique par précipitation des protéines sans que le 4-MU ne soit éliminé. Une comparaison des techniques utilisant un arrêt de la réaction enzymatique par une solution alcaline ou une précipitation par l'éthanol a été réalisée. Les activités enzymatiques étaient bien corrélées chez certains témoins, en revanche chez d'autres témoins, leur activité était très diminuée - jusqu'à $75 \%$ - avec l'arrêt de réaction par la solution alcaline, ce qui illustre le rôle de l'interférence (quenching de la fluorescence) et l'intérêt de la modification apportée à cette technique.

\section{Résultats préliminaires}

Dans une étude réalisée à l'Hôpital Européen Georges Pompidou (Caudron E, Zhou JY, Prognon P, Germain $D P$, données non publiées), des papiers filtres imbibés de sang total étaient disponibles pour 51 sujets dont 15 hommes hémizygotes, 12 femmes hétérozygotes et 24 témoins. Tous les hémizygotes présentaient une activité en $\alpha$-gal A réduite par rapport aux valeurs normales $(0,2 \pm 0,2[0-0,8]$ et $7,0 \pm 1,9[3,7-10,5] \mu \mathrm{mol} / \mathrm{h} / \mathrm{l}$ de sang respectivement) (Figure 1$)$. En revanche, seules 9 hétérozygotes sur $12(75 \%)$ des hétérozygotes avaient une activité réduite $(3,1 \pm 2,8[0-9,8] \mu \mathrm{mol} / \mathrm{h} / \mathrm{l})$ et purent être dépistées par cette technique, ce qui correspond à la sensibilité la plus élevée rapportée jusqu'à présent dans la littérature pour le dépistage des hétérozygotes par enzymologie.

En conclusion, nous avons confirmé dans ce travail I'intérêt du dosage enzymatique de l' $\alpha$-galactosidase $A$ à partir d'une goutte de sang total déposée sur papier 
filtre. La modification que nous avons apporté ici à la technique originale [1] que nous avons décrite et consistant en un arrêt de la réaction enzymatique par précipitation à l'éthanol permettant d'éliminer l'hémoglobine a permis de s'affranchir de l'interférence sur la fluorescence (quenching) imputable au noyau tétrapyrrolique de l'hème. Dans ces conditions, la sensibilité du test de dépistage sur papier filtre était de $75 \%$, supérieur au pourcentage le plus élevé rapporté jusqu'à présent dans la littérature [3]. $\diamond$

\section{SUMMARY}

Recent advances of Fabry disease screening for at risk population

An experience in screening of Fabry disease in population at risk by measuremnt of alpha-galactosidase is described. The enzymatic diagnosis in dried blood spots on filter paper is performed on 51 subjects, 15 hemizygous men, 12 heterozygous women and 24 controls. Only 9 of these 12 hererozygous women $(75 \%)$ present a reduced activity of alpha-galactosidase $A . \diamond$

\section{RéFÉRENCES}

1. Chamoles NA, Blanco M, Gaggioli D. Fabry disease : enzymatic diagnosis in dried blood spots on filter paper. Clin Chim Acta 2001 ; $308 ; 195-6$.

2. Germain DP, Poenaru L. Fabry disease : identification of novel alpha-galactosidase A mutations and molecular carrier detection by use of fluorescent chemical cleavage of mismatches. Biochem Biophys Res Commun 1999; 257 : 708-13.

3. Linthorst GE, Vedder AC, Aerts JMFG, Hollak CEM. Screening for Fabry disease using whole blood spot fails to identify one-third of female carriers. Clin Chim Acta $2005 ; 353: 201-3$

4. Mayes JS, Scheerer JB, Sifers RN, Donaldson ML. Differential assay for lysosomal alphagalactosidases in human tissues and its application to Fabry disease. Clin Chim Acta 1981 ; $112: 247-51$

5. Poeppl AG, Murray GJ, Medin JA. Enhanced filter paper enzyme assay for high-throughput population screening for Fabry disease. Anal Biochem $2005 ; 337$ : 161-3.

\section{TIRÉS À PART}

દ. Caudron 